\title{
Diversidad de microalgas y cianobacterias en muestras provenientes de diferentes provincias del Ecuador, destinadas a una colección de cultivos
}

\author{
Ever Morales ${ }^{1}$, Verónica Luna ${ }^{2}$, Lucía Navarro², Vismeli Santana ${ }^{2}$, Ana Gordillo² y \\ Andrés Arévalo \\ ${ }^{1}$ Proyecto PROMETEO-SENESCYT. \\ ${ }^{2}$ Escuela de Bioanálisis, Pontificia Universidad Católica del Ecuador, Quito, Ecuador. \\ ${ }^{3}$ Unidad de Biología, Centro de Ciencias, Universidad Central del Ecuador \\ evermster@gmail.com
}

Recibido: 24, 06, 2013; aceptado: 17, 09, 2013

RESUMEN.- El presente trabajo conforma un estudio sobre diversidad, identificación y aislamiento de microalgas y cianobacterias a partir de muestras de aguas continentales, termales, salobres y lodos; colectadas en 74 sitios de 25 sectores de las provincias del Carchi, Guayas, Imbabura, Pichincha y Santa Elena (Ecuador), entre octubre 2011 y abril 2012, con la finalidad de poder determinar en dichas muestras, su diversidad inicial, sucesión de estos microorganismos y la estabilidad de los consorcios entre microalgas y cianobacterias durante la fase de selección de muestras y de aislamiento para el establecimiento de una colección in vitro de cultivos de microorganismos fotosintéticos para la Escuela de Bioanálisis de la Pontificia Universidad Católica del Ecuador. De 194 muestras evaluadas, fueron aislados el $43.81 \%$ de microalgas y cianobacterias; donde los taxones más susceptibles de ser aislados son: Chlorella, Desmodesmus y Leptolyngbya. En cambio, se mantuvo el $56.19 \%$ como consorcios o cultivos mixtos de microalgas y cianobacterias. De estos, se cuantificaron 14 cultivos solo con cianobacterias; 40 solo entre microalgas y 55 mixtos (31 y 18, taxa de microalgas y cianobacterias; respectivamente). Los taxa más frecuentes en los consorcios microalga-microalga fueron: Chlorella sp.>Desmodesmus sp. >Navicula sp.; en los cianobacteria-cianobacteria: Leptolyngbya sp.> Calothrix sp.> Nostoc sp. y en los microalga-cianobacteria: Leptolyngbya sp.>Navicula sp.>Chlorella sp.> Desmodesmus sp.> Calothrix sp. Las muestras obtenidas en la laguna "El Salado", San Gabriel, Carchi presentaron la mayor diversidad. Mientras que, las colectadas en "Aguas Hediondas", Tufiño, Carchi mostraron exclusividad de Dictyosphaerium sp., Microspora sp., y Mougeotia sp. La cianobacteria Leptolyngbya sp., refleja ser altamente competitiva en cultivos envejecidos y deficientes en nutrientes. Se recomienda ingresar todos los consorcios a las colecciones, dada su importancia como fuentes de biodiversidad y para estudios de fisioecología, biotecnología ambiental y agrícola.

PALABRAS CLAVES: cianobacteria, colección de cultivos, consorcio, Ecuador, microalga 
ABSTRACT.- We conducted a study on diversity, identification and isolation of microalgae and cyanobacteria from inland waters, springs, brackish water and slurries collected at 74 sites in five province: Carchi, Guayas, Imbabura, Pichincha and Santa Elena (Ecuador), between October 2011 and April 2012. The samples were characterized by initial diversity, succession of these microorganisms, and the stability of microalgae and cyanobacteria consortia during the sample selection and isolation process. These isolates were used for the establishment of a collection of in vitro cultures of photosynthetic microorganisms for the School of Bioanalysis of the Pontificia Universidad Católica del Ecuador. From the 194 samples tested, $43.81 \%$ of the isolates were single species of microalgae and cyanobacteria. The taxa more likely to be isolated were the genera, Chlorella, Scenedesmus and Leptolyngbya. In contrast, $56.19 \%$ of the isolates remained as consortia or mixed cultures of microalgae and cyanobacteria. Of these, 14 cultures were only cyanobacteria, 40 were microalgae and 55 were mixed cultures ( 31 and 18 taxa of microalgae and cyanobacteria, respectively). The most common genera of microalgae in the microalgae consortia were, Chlorella sp. $>$ Scenedesmus sp. $>$ Navicula sp. The most common genera in the cyanobacteria consortia were Leptolyngbya sp. > Calothrix sp. $>$ Nostoc sp. The most common genera in the microalgaecyanobacteria consortia were Leptolyngbya sp. > Navicula sp. > Chlorella sp. > Scenedesmus sp. > Calothrix sp. The samples from Laguna "El Salado", San Gabriel, Carchi had the highest diversity. While, those collected in Aguas Hediondas Tufiño, Carchi contained only Dictyosphaerium sp., Microspora sp. and Mougeotia sp. The cyanobacterium Leptolyngbya sp. was highly competitive and characterized aged cultures and nutrient deficiencies. It is recommended that all consortia be entered into culture collections because of their importance as sources of biodiversity. These consortia can also be important in physiological, ecology and environmental studies and development of techniques for agricultural biotechnology.

KEYWORDS: cyanobacteria, consortium, culture collection, Ecuador, microalgae

\section{INTRODUCCIÓN}

La importancia que presentan los microorganismos fotosintéticos como productores primarios, fuentes de compuestos de interés económico, agentes depuradores de aguas residuales, de suelos contaminados, complemento alimenticio, o como bioindicadores de calidad de aguas, conlleva a establecer estrategias de su conservación ex situ (Lascuráin et al., 2009), mediante la creación de colecciones in vivo. Estas, constituyen recursos genéticos para estudios de biodiversidad y de biotecnología (Friedl y Lorenz, 2012; GBIF, 2009; Ortega, 2009). Así como para estudios integrados de bioprospección (Castree, 2003), relacionados con aislamiento, identificación y cultivo, especialmente de microorganismos procedentes de ambientes extremófilos, subtropicales, tropicales, marinos y de otros ambientes. Tales estudios representaran una base 
para la investigación de sus características morfólogicas, moleculares, metabólicas y su posible utilización para los diversos fines indicados. $\mathrm{Al}$ respecto, el Banco Español de Algas (BEA), con sede en la Universidad de Las Palmas de Gran Canaria de España, contempla permanentemente estudios sobre aislamiento, conservación y evaluación de cepas para su aplicación en biotecnología relacionadas con iniciativas científicas e industriales para la producción de biocombustibles y captación de $\mathrm{CO}_{2}$ (García, 2011; Banco Español de Algas, 2008).

Estos lineamientos reflejan la necesidad de creación de las colecciones vivas de microalgas y cianobacterias, para investigación básica en taxonomía, ecología, genética, estructura y biogeografía (Mora, 2010; Delgadillo et al., 2009; López-Adrián y Barrientos-Medina, 2005). A través de una colección se podrá ofrecer una amplia colección de microalgas y cianobacterias que permita la selección de cepas como fuente de biocombustible, de compuestos con actividad biológica, para acuicultura, biofertilizantes, etc.

Entre otros aspectos, los bancos de germoplasma, destinados al mantenimiento de cepas aisladas de ambientes naturales permiten tener accesibles líneas genéticas particulares de especies o grupos taxonómicos de interés para su estudio, comercialización o reintroducción a la naturaleza en forma de poblaciones vivas (Fernández-Concha et al., 2004; Buitrago et al., 1999). No obstante, para la preservación del germoplasma estos microorganismos fotosintéticos como para todas las colecciones de heterótrofos, se requiere la gran labor del curador que asegure la reserva genética de estos organismos y cuyas técnicas van desde un crecimiento continuo hasta la aplicación de estrategias que reducen la velocidad del metabolismo mediante métodos de criopreservación o secado por congelamiento (FernándezConcha et al., 2004).

En cuanto a las actividades iniciales para la creación o adición de cepas a una colección, es indispensable el conocimiento y entrenamiento de las técnicas de colección de muestras, que permitan la preservación y adecuada manipulación de las mismas, para su posterior análisis y determinación taxonómica (Montes de Oca et al., 2008; Stein, 1973). Posteriormente, se pasa a la fase de aislamiento mediante el uso de diversos medios de cultivo según sea la procedencia y tipo de microalga y cianobacteria. Normalmente las instituciones o laboratorios de investigación conservan colecciones acreditadas de cultivos, desde donde se pueden obtener las cepas unialgales y en medios selectivos o genéricos para las especies marinas o dulceacuícolas.

Entre las colecciones más importantes a nivel mundial, se reporta la colección de la Universidad de Texas de Estados Unidos (Starr, 2010); en Australia se cuenta con la Colección Nacional de cultivos de algas de Australia (ANACC-CSIRO). Mientras que, en Portugal se destaca la Algoteca de la Universidad de Coimbra (Santos, 
2008) y en Francia se mantiene el cepario de cianobacterias del Instituto Pasteur (Billard, 1987). En relación con latinoamérica, se tiene la colección de microalgas del Centro de Investigaciones Biológicas del noroeste de México (Arredondo, 1997); en Argentina la de la Universidad Nacional de la Patagonia "San Juan Bosco" (Albarracín et al., 2009) y en Chile la colección de microalgas de la Universidad de Concepción (González et al., 2008).

Se resalta en el presente trabajo la investigación realizada en el laboratorio de Ficología de la Pontificia Universidad Católica del Ecuador (PUCE). Se la inició con la colecta de muestras en diferentes sectores geográficos del Ecuador, identificación de cepas y elaboración de un registro de las mismas, mediante una base de datos, así como también, la selección de áreas geográficas para bioprospección de microalgas y cianobacterias de interés en biotecnología ambiental, biomedicina y agroalimentación.

En relación con estudio de monitoreo de muestras colectadas en los diferentes ambientes seleccionados para su posterior aislamiento, no se tienen reportes, ni información en artículos científicos sobre colecciones de microalgas y cianobacterias existentes en el Ecuador. Tampoco, se han descrito reportes sobre los diferentes taxa presentes en una muestra original y como las condiciones de laboratorio pueden modular su población y permanencia durante el proceso de aislamiento. Aun cuando el curador debe tratar de aislar el mayor número posible de especies, en función de los medios de cultivos utilizados, condiciones de temperatura, nutrientes e iluminación.

Por ello, el presente trabajo pretende demostrar la importancia de registrar la biodiversidad de microalgas y de cianobacterias procedentes de distintos ambientes geográficos del Ecuador, así como, la necesidad de reportar la existencia de los taxa observados, y las microalgas y cianobacterias susceptibles de ser conservadas en consorcios nativos procedentes de los sitios de colecta. Además, se hace referencia a la importancia de promover las colecciones de microalgas como línea base para investigación básica y aplicada a biotecnología, ecofisiología y taxonomía en el Ecuador.

\section{MATERIALES Y MÉTODOS}

Sitios de muestreos.- Se realizaron muestreos de campo en las provincias del Carchi, Guayas, Imbabura, Pichincha y Santa Elena (Ecuador). La Tabla 1 señala las localidades y el número de muestras colectadas por estaciones. 


\section{Tabla 1}

Sitios de muestreo (provincias Santa Elena, Guayas, Carchi e Imbabura, Ecuador)

\begin{tabular}{|c|c|}
\hline PROVINCIA & Localidad \\
\hline CARCHI & $\begin{array}{l}\text { Estación1: Carretera asfaltada vía San Gabriel, después } \\
\text { de la Gruta de la Paz (3) } \\
\text { Estación 2: Gruta de la Virgen, Balneario (3) } \\
\text { Estación 3: Laguna El Salado, San Gabriel (2) } \\
\text { Estación 4: Termas Aguas Hediondas (3) } \\
\text { Estación 5: Termas-Placa Proyecto Geotérmico (3) }\end{array}$ \\
\hline GUAYAS & $\begin{array}{l}\text { Estación 1: Parque del Lago (1) } \\
\text { Estación 2: Bosque de Manglar, Puerto Hondo (1) } \\
\text { Estación 3: Recinto San Gabriel, Vía Daule (2) } \\
\text { Estación 4: Recinto Correntoso (2) }\end{array}$ \\
\hline IMBABURA & $\begin{array}{l}\text { Estación 1: Dique, San Antonio de Ibarra (2) } \\
\text { Estación 2: Lago San Pablo (5) } \\
\text { Estación 3: Laguna Yahuarcocha (3) } \\
\text { Estación 4: Mojanda (2) } \\
\text { Estación 5: Termas de Chachimbiro (3) }\end{array}$ \\
\hline PICHINCHA & $\begin{array}{l}\text { Estación 1: Parque Metropolitano (2) } \\
\text { Estación 2: Jardín Botánico (11) } \\
\text { Estación 3: Amaguaña (2) } \\
\text { Estación 4: Parque Cayambe-Coca, Papallacta (6) } \\
\text { Estación 5: Parque La Moya (1) }\end{array}$ \\
\hline SANTA ELENA & $\begin{array}{l}\text { Estación 1: Baños termales de San Vicente (5) } \\
\text { Estación 2: CENAIN (piscina de cultivo con tilapia (2) } \\
\text { Estación 3: Comuna El Azúcar, Zapotal (2) } \\
\text { Estación 4: La Chocolatera, Salinas (3) } \\
\text { Estación 5: Piscinas de sal de Monteverde (2) }\end{array}$ \\
\hline
\end{tabular}

Entre paréntesis se indica el número de sitios muestreados en cada sector.

Toma de muestra.- Las muestras fueron colectadas en frascos de vidrio, envases plásticos y en bolsas de polietileno etiquetados a partir de aguas, lodos, sedimentos y de superficie de rocas. Para la colecta de fitoplancton en la laguna de Yahuarcocha y en el lago San Pablo, Imbabura (Ecuador), se utilizó una red de fitoplancton de $22 \mu \mathrm{m}$. Las muestras de lodos y sedimentos fueron colectadas manualmente con envases de plástico para muestras biológicas y con espátula. Las comunidades de microalgas crecidas sobre rocas y piedras fueron colectadas mediante raspados con cepillo en bolsas de polietileno o envases plásticos y luego transportadas hasta el laboratorio. Cada una delas muestras en los diferentes sitios fueron extraídas por duplicado (Serrano et al., 2004).

Trabajo de laboratorio.- Para cada muestra se consignaron los caracteres del hábitat, localidad, fecha, número del colector y pH (Vergara, 2009; González et al., 1995). A las muestras colectadas en zonas marinas se les determinó la salinidad 
mediante el uso de un refractómetro. A partir de cada muestra original, se tomaron tres submuestras para su observación microscópica e identificación taxonómica. Para Cyanoprokaryota se utilizó la clave de Anagnostidis y Komárek (1988) y de Komárek y Anagnostidis (1989, 2005); para Chlorophyta la de Bourrelly (1981) y Eaton et al., 1995 y las de Bacillariophycea según Krammer y Lange-Bertalo (1991) y Rumrich et al. 2000.

Siembra de muestras en medios de cultivos líquidos y sólidos.- Las muestras de fitoplancton fueron resuspendidas en los medios de cultivo $\mathrm{F} / 2$ y fertilizante comercial Nitrofoska cuando las muestras estaban muy enriquecidas con diatomeas. En cambio, se utilizaron medio de cultivo BG11 completo y BG11 ${ }_{0}$ sin nitrógeno para las muestras con una población significativa de cianobacterias sin heterocitos o con heterocitos, respectivamente (Serrano et al., 2004).

Para el caso de las muestras marinas o de aguas termales salobres se utilizaron los medios BG11, F/2 y fertilizante foliar Nitrofoska con ClNa al $3.5 \%$. Luego, las muestras eran transferidas tanto a medios líquidos no aireados, como a medios sólidos para dar inicio al aislamiento tanto de microalgas como de cianobacterias. Los cultivos en medio líquido BG11 completo, BG11, Nitrofoska se mantuvieron en volúmenes de $10 \mathrm{ml}$ en tubos de ensayos no herméticamente tapados. Todos fueron colocados bajo iluminación artificial continua y con lámparas fluorescentes a una irradiancia de $78 \mu \mathrm{mol}$ quanta. $\mathrm{m}^{-1} \cdot \mathrm{s}^{-1}$ en condiciones de laboratorio.

Cada 15 días fueron monitoreados en cultivos, los cambios en cuanto a la población y existencia de taxa. Esto se realizaba sobre todo con los tubos de ensayo en donde aún permanecían los consorcios o mezclas tanto de microalgas como de cianobacterias. La densidad de población se determinó por observación al microscopio por recuento en cámara de Neubauer. Las muestras colonizadas por micelio o aquellas que por efecto del medio de cultivo y/o de las condiciones del laboratorio perdían viabilidad, fueron descartadas.

\section{RESULTADOS}

A partir de las observaciones realizadas a las muestras colectadas se reportan las microalgas y cianobacterias que permanecen asociadas en consorcios y las aisladas en los diferentes medios de cultivos; como también las que se mantienen en las muestras originales. Este reporte, permitió establecer la estabilidad de las poblaciones existentes en los microambientes estudiados con las cuales han sido inoculadas en los medios de cultivos.

Las localidades con mayor riqueza específica de microalgas fueron la Comuna "El Azúcar", Zapotal, "Parque La Moya", "Recinto Correntoso", Vía Daule, "Amaguaña", laguna "Yahuarcocha" y lago "San Pablo"; siendo la laguna "El Salado", San Gabriel, Carchi la más representativa con 14 taxa, de los cuales 13 corresponden a las diatomeas: Gomphonema sp., Navicula 
sp, Niztschia closterium y Synedra sp.; a laboratorio, la riqueza específica se redujo a las clorofitas: Spirogyra sp., Staurastrum Navicula sp., y Gomphonema sp., pero con la sp., Lagerheimia citriformis, Kirchneriella adición de Pseudanabaena sp. y Leptolyngbya sp., Tetraedron sp., Oedogonium sp., sp.; lo cual deduce que las clorofitas parecen Desmodesmus sp., y una no identificada en ser susceptibles a cambios ambientales en estado palmeloide y a las cianobacterias: condiciones de laboratorio, ya que ninguna Oscillatoria sp. y Microcystis sp. (Tabla 2a). fue observada en las evaluaciones realizadas Al término de dos meses en condiciones de con posterioridad.

\section{Tabla 2a}

Registro de microalgas y cianobacterias (abundancia y observaciones) en muestras colectadas en varios sitios sectores de la provincia del Carchi

\begin{tabular}{|c|c|c|c|}
\hline $\begin{array}{l}\text { Procedencia } \\
\text { muestra }\end{array}$ & $\begin{array}{l}\text { Microalga/ } \\
\text { cianobacteria }\end{array}$ & Abundancia & Observaciones \\
\hline 1/Gruta de la Paz & $\begin{array}{l}\text { Navicula sp. } \\
\text { Chlorella sp. }\end{array}$ & $\begin{array}{l}++++ \\
+\end{array}$ & $\begin{array}{l}\text { Raspado de rocas. } \\
\text { Medio F/ } 2\end{array}$ \\
\hline 2a/Gruta de la Paz & $\begin{array}{l}\text { Navicula sp. } \\
\text { Leptolyngbya sp. }\end{array}$ & $\begin{array}{l}+++ \\
+\end{array}$ & $\begin{array}{l}\text { Raspado de rocas. Medio } \\
\text { F/2 }\end{array}$ \\
\hline 2b/Gruta de la Paz & Diatoma sp. & & $\begin{array}{l}\text { Poca presencia de } \\
\text { microalgas. }\end{array}$ \\
\hline $\begin{array}{l}\text { 3/Gruta de la Paz. } \\
\text { camino hacia las } \\
\text { piscinas }\end{array}$ & $\begin{array}{l}\text { Anabaena sp. } \\
\text { Diatomea pennada } \\
\text { Navícula sp. }\end{array}$ & $\begin{array}{l}+++++++ \\
++ \\
+\end{array}$ & $\begin{array}{l}\text { Numerosos acinetos. } \\
\text { Siembra en medios BG11 y } \\
\text { F/2. }\end{array}$ \\
\hline $\begin{array}{l}\text { 4/Gruta de la Paz. } \\
\text { Camino hacia las } \\
\text { piscinas, caída de agua. } \\
\text { Suelo con briofitas }\end{array}$ & $\begin{array}{l}\text { Anabaena sp. } \\
\text { Navicula sp. }\end{array}$ & $\begin{array}{l}+++++ \\
+\end{array}$ & Medio BG11 \\
\hline $\begin{array}{l}\text { 5/Gruta de la Paz. Orilla } \\
\text { del río. }\end{array}$ & $\begin{array}{l}\text { Phormidium sp. } \\
\text { Oscillatoria sp. } \\
\text { Lyngbya sp. }\end{array}$ & $\begin{array}{l}+++++ \\
++ \\
+\end{array}$ & $\begin{array}{l}\text { Siembra en medio sólido } \\
\text { BG11. }\end{array}$ \\
\hline $\begin{array}{l}\text { 6/ Carretera asfaltada } \\
\text { hacia San Gabriel-Km 3, } \\
\text { después de la Gruta de } \\
\text { la Virgen. }\end{array}$ & $\begin{array}{l}\text { Closterium sp. } \\
\text { Navícula sp. } 1 \\
\text { Navicula sp. } 2 \\
\text { Anabaena azollae } \\
\text { Cosmarium sp. } \\
\text { Pandorina sp. }\end{array}$ & $\begin{array}{l}+++ \\
+++ \\
+ \\
+ \\
+ \\
+\end{array}$ & $\begin{array}{l}\text { Agua estancada cubierta } \\
\text { por Azolla. Siembra en } \\
\text { BG11.Presencia de } \\
\text { Closterium sp. con } \\
\text { cygospora. }\end{array}$ \\
\hline $\begin{array}{l}\text { 7/Laguna El Salado-San } \\
\text { Gabriel-Muelle }\end{array}$ & $\begin{array}{l}\text { Spirogyra sp. } \\
\text { Gomphonema sp. } \\
\text { Navícula sp. } \\
\text { Nitzschia closterium } \\
\text { Staurastrum sp. } \\
\text { Lagerheimia citriformis } \\
\text { Kirchneriella sp. } \\
\text { Tetraedron } \mathrm{sp} . \\
\text { Oedogonium } \mathrm{sp} . \\
\text { Desmodesmus } \mathrm{sp} . \\
\text { Oscillatoria } \mathrm{sp} . \\
\text { Microcystis sp. } \\
\text { Synedra sp. }\end{array}$ & $\begin{array}{l}\text { ++++++++++ } \\
++++++++ \\
++ \\
++ \\
+ \\
++ \\
++ \\
+ \\
+ \\
+ \\
+ \\
+ \\
+\end{array}$ & $\begin{array}{l}\text { Predominio de Spirogyra } \\
\text { sp. } \\
\text { Muestra con mayor } \\
\text { diversidad de microalgas. } \\
\text { Siembra en medio sólido y } \\
\text { líquido con fertilizante } \\
\text { Nitrofoska. }\end{array}$ \\
\hline $\begin{array}{l}8 / \text { charca orilla carretera } \\
\text { frente a la laguna } \mathrm{El}\end{array}$ & $\begin{array}{l}\text { Spirogyra sp. } \\
\text { Anabaena sp. } \\
\text { Navícula sp. }\end{array}$ & $\begin{array}{l}+++++ \\
+++ \\
++\end{array}$ & $\begin{array}{l}\text { Siembra en medio líquido } \\
\text { NF y en medio sólido } \\
\text { BG11. Adición de extracto } \\
\text { de levadura. }\end{array}$ \\
\hline
\end{tabular}


La Tabla $2 \mathrm{~b}$ presenta los microorganis- microalga colonial Dictyosphaerium sp., y mos hallados a partir de las muestras colec- acompañada de una menor población de tadas del balneario de "Aguas Hediondas", Anabaena sp., y Cosmarium sp.. En relación Tufiño. En una de ellas, solo se observó con la presencia de Dictyosphaerium sp., un crecimiento exclusivo de Ulothrix sp., poco frecuente en muestras, se caracterizó y mientras que en otra muestra del sector por mantener su predominio durante cuatro sorprende la presencia casi exclusiva de la meses en la muestra original.

Tabla $2 b$

Registro de microalgas y cianobacterias (abundancia y observaciones) en muestras colectadas en varios sitios del Balneario de Aguas Hediondas, Tufiño, Carchi

\begin{tabular}{|c|c|c|c|}
\hline $\begin{array}{c}\text { Muestra/ } \\
\text { Procedencia }\end{array}$ & $\begin{array}{l}\text { Microalga/ } \\
\text { cianobacteria }\end{array}$ & Abundancia & Observaciones \\
\hline $\begin{array}{l}\text { 9a/Balneario de Aguas } \\
\text { Hediondas }\end{array}$ & $\begin{array}{l}\text { Crecimiento exclusivo } \\
\text { de Ulothrix sp. }\end{array}$ & ++++++++++ & $\begin{array}{l}\text { Riachuelo del } \\
\text { balneario de Agua } \\
\text { Hediondas }\end{array}$ \\
\hline $\begin{array}{l}\text { 9b/Balneario de Aguas } \\
\text { Hediondas-piscina }\end{array}$ & $\begin{array}{l}\text { Diatomeas pennadas. } \\
\text { Filamento uniseriado }\end{array}$ & $\begin{array}{l}+++++++++ \\
++\end{array}$ & $\begin{array}{l}\text { Raspado de piedra. } \\
\text { Diversidad de } \\
\text { diatomeas. } \\
\text { Medio } \mathrm{F} / 2\end{array}$ \\
\hline $\begin{array}{l}\text { 9c/Balneario de Aguas } \\
\text { Hediondas }\end{array}$ & $\begin{array}{l}\text { Dictyosphaerium sp. } \\
\text { Anabaena sp. } \\
\text { Cosmarium sp. }\end{array}$ & $\begin{array}{l}+++++++++++ \\
+++ \\
+\end{array}$ & Medio NF \\
\hline $\begin{array}{l}\text { 9d/Balneario de Aguas } \\
\text { Hediondas, Placa } \\
\text { Proyecto Geotérmico. }\end{array}$ & $\begin{array}{l}\text { Clorofitas esféricas en } \\
\text { pares en matriz } \\
\text { mucilaginosa. } \\
\text { Oocystis sp. } \\
\text { Cosmarium } \mathrm{sp} \text {. } \\
\text { Navicula } \mathrm{sp} \text {. } \\
\text { Microspora sp. } \\
\text { Nostoc sp. }\end{array}$ & $\begin{array}{l}++++++ \\
+ \\
++ \\
+ \\
++++ \\
+ \\
+\end{array}$ & $\begin{array}{l}\text { Muestra con } \\
\text { briofitas. Medio } \\
\text { sólido BG11 y } \\
\text { medio líquido NF. }\end{array}$ \\
\hline $\begin{array}{l}\text { 10a/Balneario de Aguas } \\
\text { Hediondas, Placa } \\
\text { Proyecto Geotérmico. }\end{array}$ & $\begin{array}{l}\text { Mougeotia sp. } \\
\text { Chlamydomonas sp. } \\
\text { Oocystis sp. } \\
\text { Zygnema sp. }\end{array}$ & $\begin{array}{l}++++ \\
+ \\
+ \\
+\end{array}$ & Medio líquido NF. \\
\hline $\begin{array}{l}\text { 10b/Balneario de Aguas } \\
\text { Hediondas, Placa } \\
\text { Proyecto Geotérmico. }\end{array}$ & $\begin{array}{l}\text { Closterium sp. } \\
\text { Zygnema sp. }\end{array}$ & $\begin{array}{l}\text { Predominante } \\
++\end{array}$ & $\begin{array}{l}\text { Sedimento } \\
\text { Medio líquido y } \\
\text { sólido NF. }\end{array}$ \\
\hline
\end{tabular}

Abundancia: recuento estimado de microorganismos, unicelulares, coloniales o filamentos por campo de observación al microscopio. Medio NF: fertilizante comercial NITROFOSKA. Valoración de abundancia: escasa (+), moderada (desde ++ hasta +++++ ), abundante (desde ++++++ hasta ++++++++++ ). 
De un total de 194 muestras abundanciaChlorella sp.>Desmodesmus sp.> evaluadas, se aisló el $42.27 \%$, de las cuales Leptolyngbya sp.>Chroococcus sp., con un 23 corresponden a cianobacterias y 68 a las $32 \%, 13 \%, 13 \%$ y $8 \%$, respectivamente microalgas; encontrándose por orden de (Figura 1).

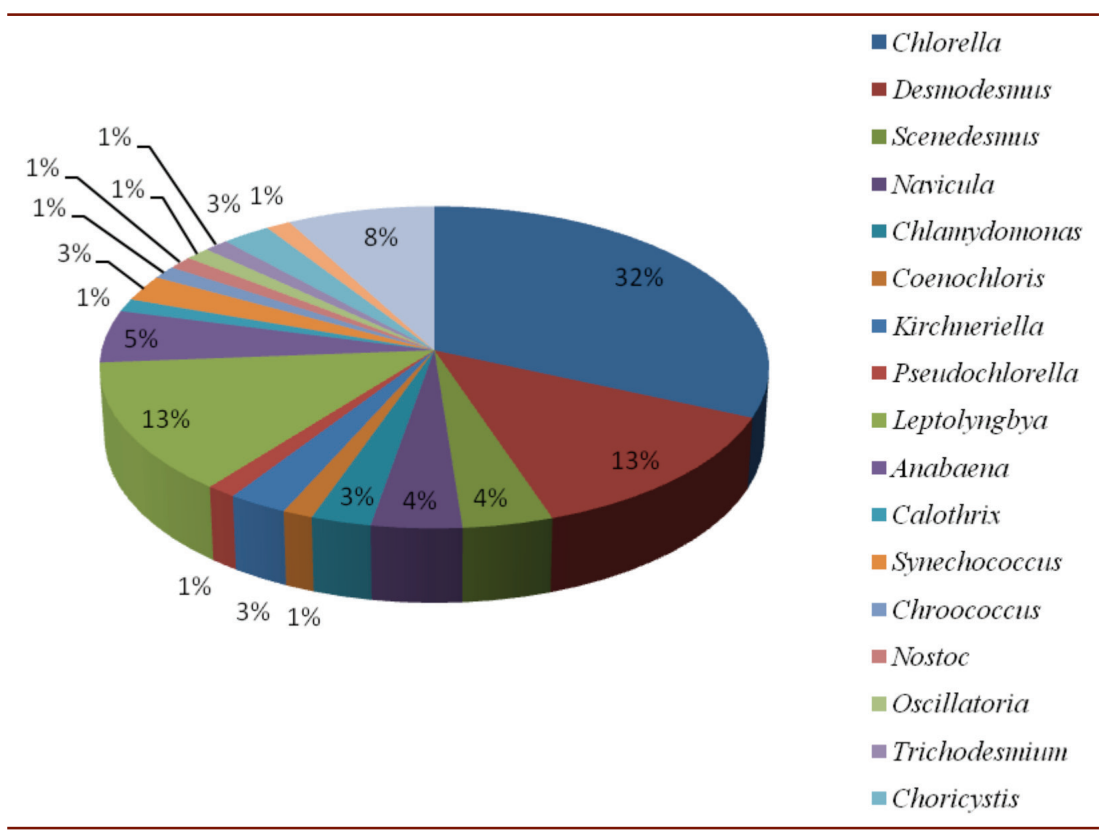

Figura 1. Porcentaje (\%) de los principales taxa de microalgas y de cianobacterias aisladas.

En cambio, los consorcios alcanzaron 53 consorcios microalga-cianobacteria, con el $57.73 \%$, identificándose 40 muestras con 31 y 19 taxa de microalga y cianobacteria, consorcios microalga-microalga (37 taxa); respectivamente (Figura 2). 12 cianobacteria-cianobacteria (6 taxa) y

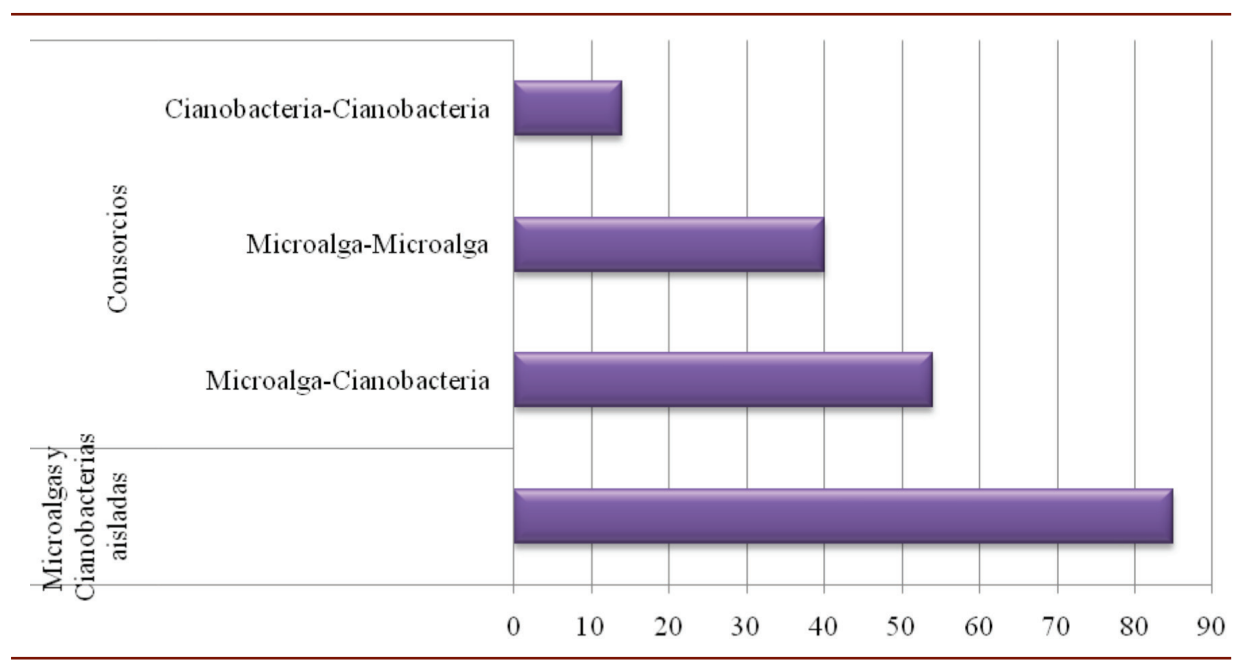

Figura 2. Número de muestras en consorcios: microalga-microalga, microalga-cianobacteria y cianobacteria-cianobacteria y de géneros de microalgas y de cianobacterias aisladas. 
De acuerdo con el número de taxa en diatomeas y por último las euglenofitas, los consorcios, las clorofitas fueron las más con $49.0 \%$; $30.0 \%, 17.5 \%$ y $3.2 \%$, abundantes, seguido de las cianobacterias, respectivamente (Figura 3).

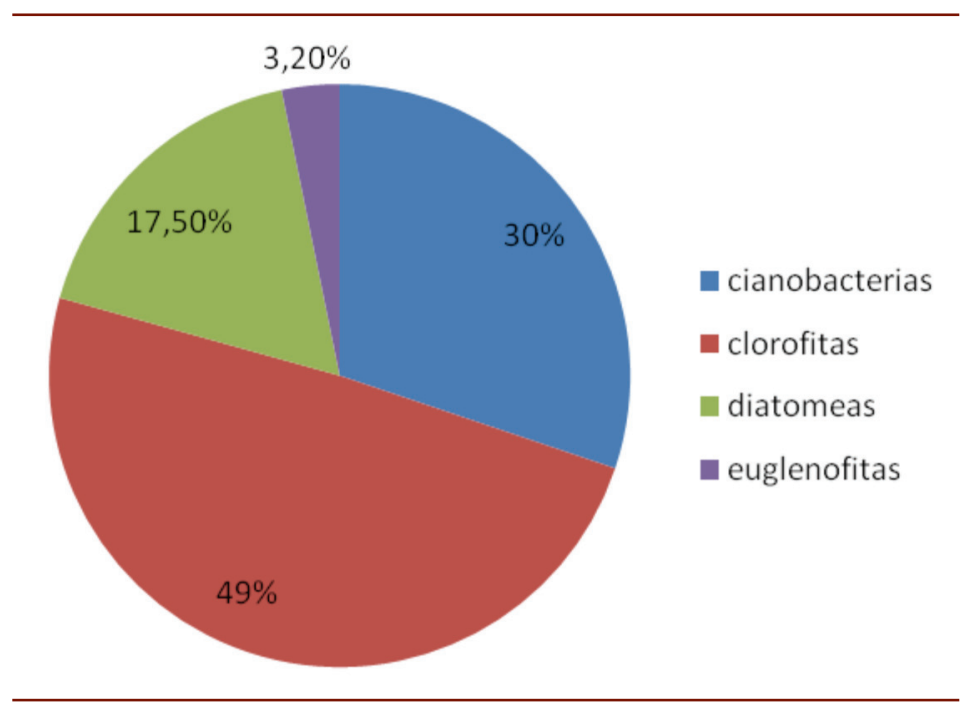

Figura 3. Porcentaje (\%) de taxa de cianobacterias, clorofitas, diatomeas y euglenofitas presentes en los consorcios.

En los consorcios entre microalgas, las En cambio, en los consorcios entre cianobacmayores frecuencias de aparición estuvo en terias, se distinguieron Leptolyngbya sp. y el siguiente orden: Chlorella sp.> Navicula Calothrix sp. (Figura 5) sp.> Desmodesmus sp. (Figura 4).

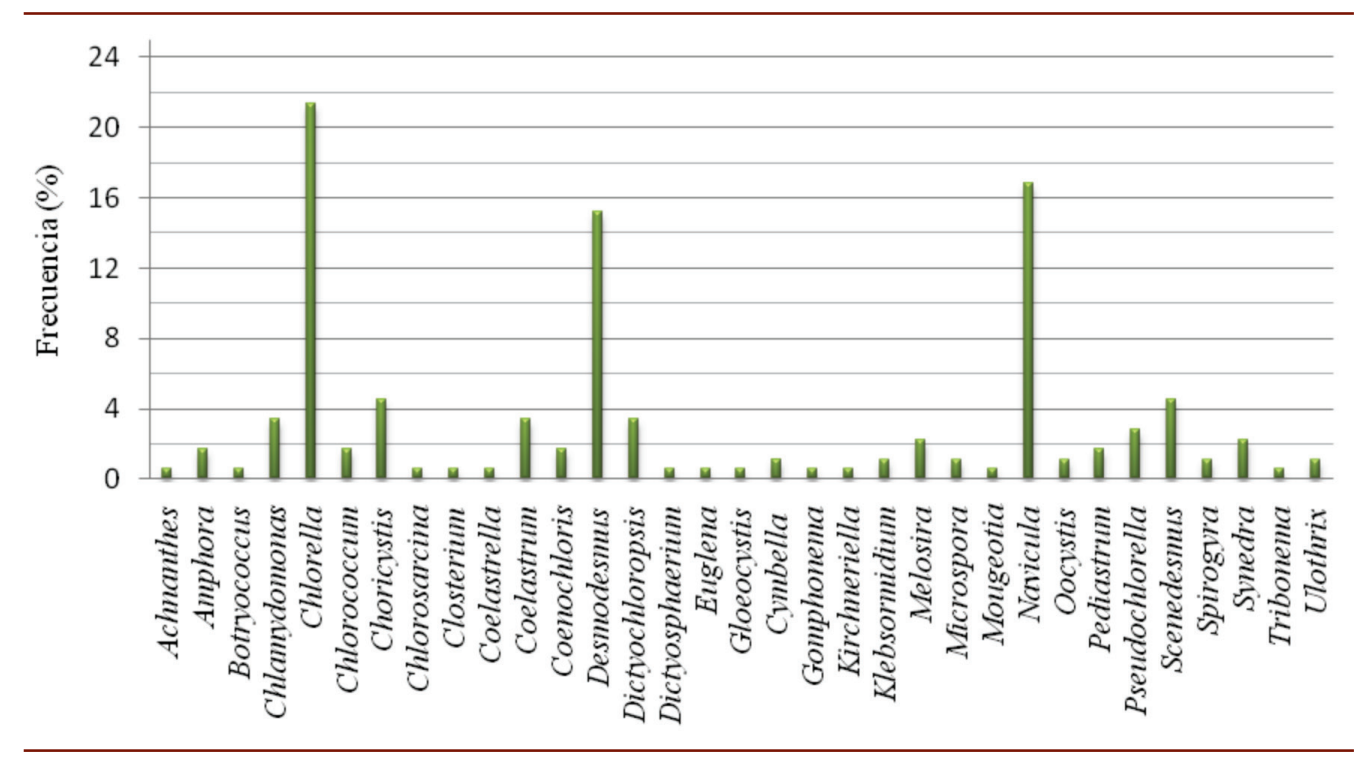

Figura 4. Diversidad y frecuencia (\%) de taxa presentes en consorcio entre microalgas. 


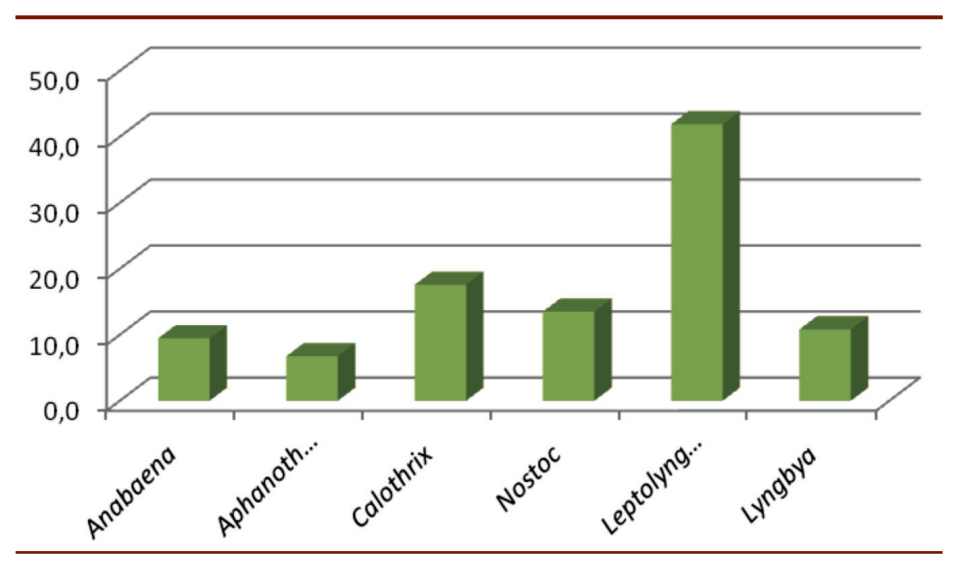

Figura 5. Diversidad y frecuencia de taxa presentes en consorcios de cianobacterias.

Al evaluar la diversidad y frecuencia otra, parte se observó una baja frecuencia de géneros en los consorcios microalga- de los taxa: Eunotia sp., Achnanthes sp., cianobacteria, se destacó una alta Selenastrum sp., Closterium sp., Cosmarium presencia de Chlorella sp., Desmosdesmus sp., Monoraphidum sp., Gloeocystis sp., sp. y Leptolyngbya sp., lo cual confirma el Mougeotia sp., Spirogyra sp., Microcoleus predomino de estos microorganismos en sp., Trichodesmium sp. y Aphanizomenon sp.; todas las muestras evaluadas, e incluso en los cuales se incluyen en la Figura 6, como las aisladas y mantenidas en colección. Por "otros taxa".

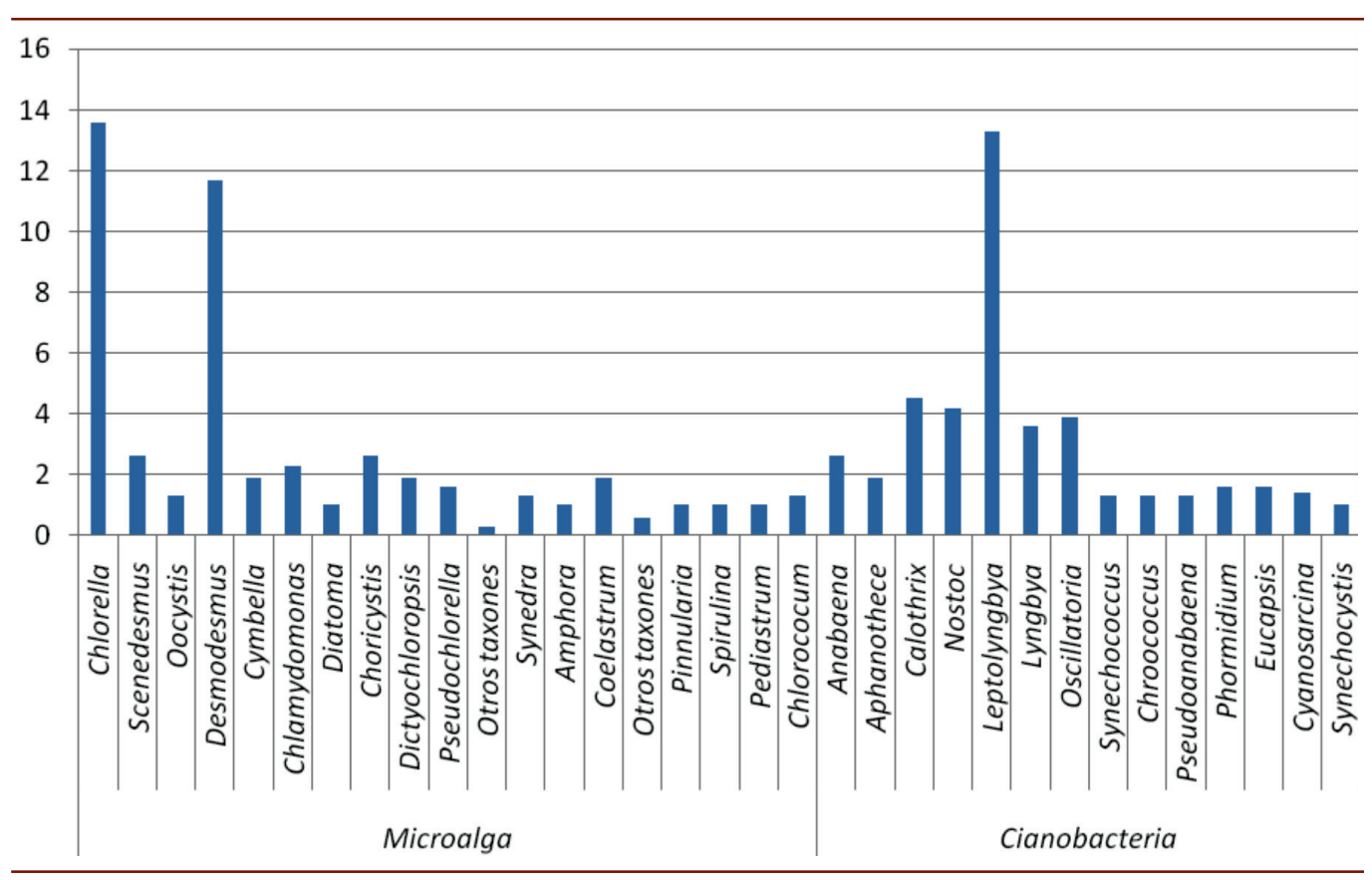

Figura 6. Diversidad y frecuencia de taxa presentes en consorcios mixtos microalgas- cianobacterias. Otros taxa: Eunotia sp., Selenastrum, sp. Cosmarium sp., Monoraphidum sp., Mougeotia sp., Gloecosytis sp., Spirogyra sp., Closterium sp., Achnanthes sp., Microcoleus sp., Trichodesmium sp. y Aphanizomenon sp. 
Se destaca la presencia de significativo en relación con Anabaena sp., Dictyosphaerium sp. (Figura 7), encontrada y Cosmarium sp., en consorcios microalgaen el sector del balneario de aguas cianobacteria, durante cuatro meses en termales de "Aguas Hediondas", Carchi, condiciones de laboratorio. debido a que mantuvo con un predomino

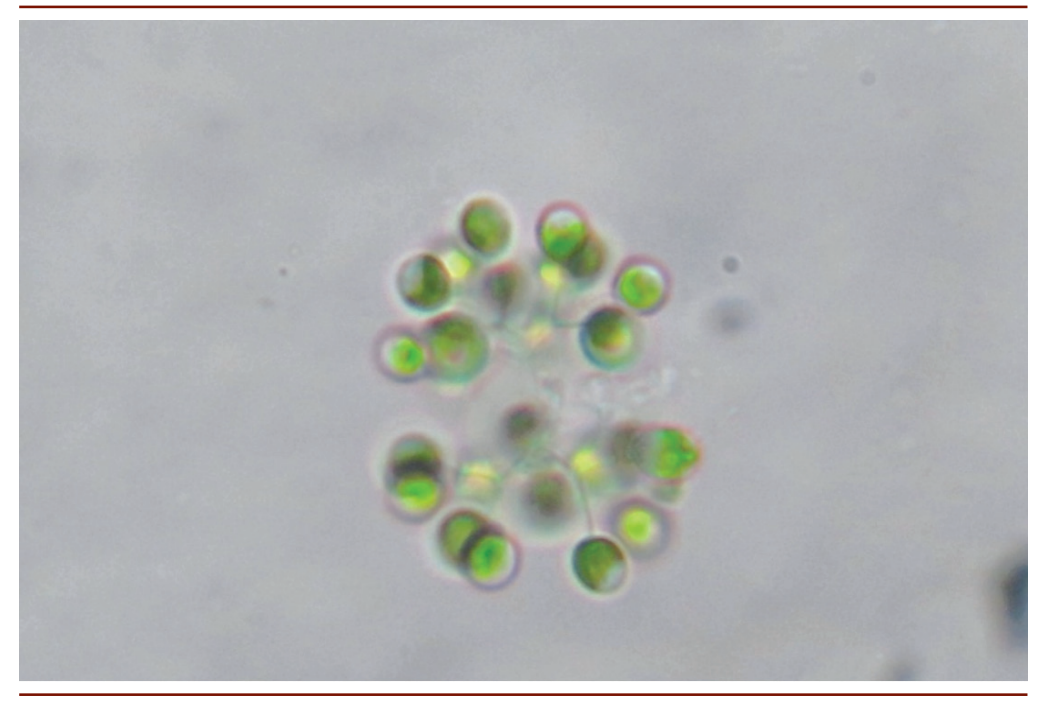

Dictyosphaerium sp. (40x)

Figura 7. Dictyosphaerium sp., Nägeli (1849). Observado solo en el sector de "Aguas Hediondas", Tufiño, provincia del Carchi, Ecuador.

Dyctiosphaerium sp. Nägeli (1849), se caracteriza por su organización colonial esférica o irregular, envuelta por una matriz mucilaginosa, con 4-64 células esféricas u ovales conectadas por filamentos gelatinizados, las cuales forman una estructura cruciforme de ramificación dicotómica o tetracotómica y dispuestas perpendicularmente a la superficie de la colonia. Puede crecer en una variedad de hábitats de agua dulce, así como en embalses eutróficos y estanques (Krienitz et al., 2012; Krienitz et al., 2010).

Microspora, Thuret (1850), (Figura 8) también fue observada en el mismo sector de las aguas termales de "Aguas
Hediondas", provincia del Carchi y se caracteriza por presentar filamentos cilíndricos uniseriados y no ramificados. $\mathrm{Su}$ carácter taxonómico sobresaliente, corresponde a un engrosamiento diferencial de la pared celular, de modo que su parte próxima al septo transversal es siempre más gruesa y se va adelgazando hacia la porción media de la célula. En algunas ocasiones ocurre la ruptura del filamento en la parte media formándose así, fragmentos de pared celular con forma de H. Están distribuidas en habitats acuáticos, particularmente entre algas filamentosas en arroyos, riachuelos, acequias y efluentes de minería con $\mathrm{pH}$ entre 3-6 (Guiry, 2013). 


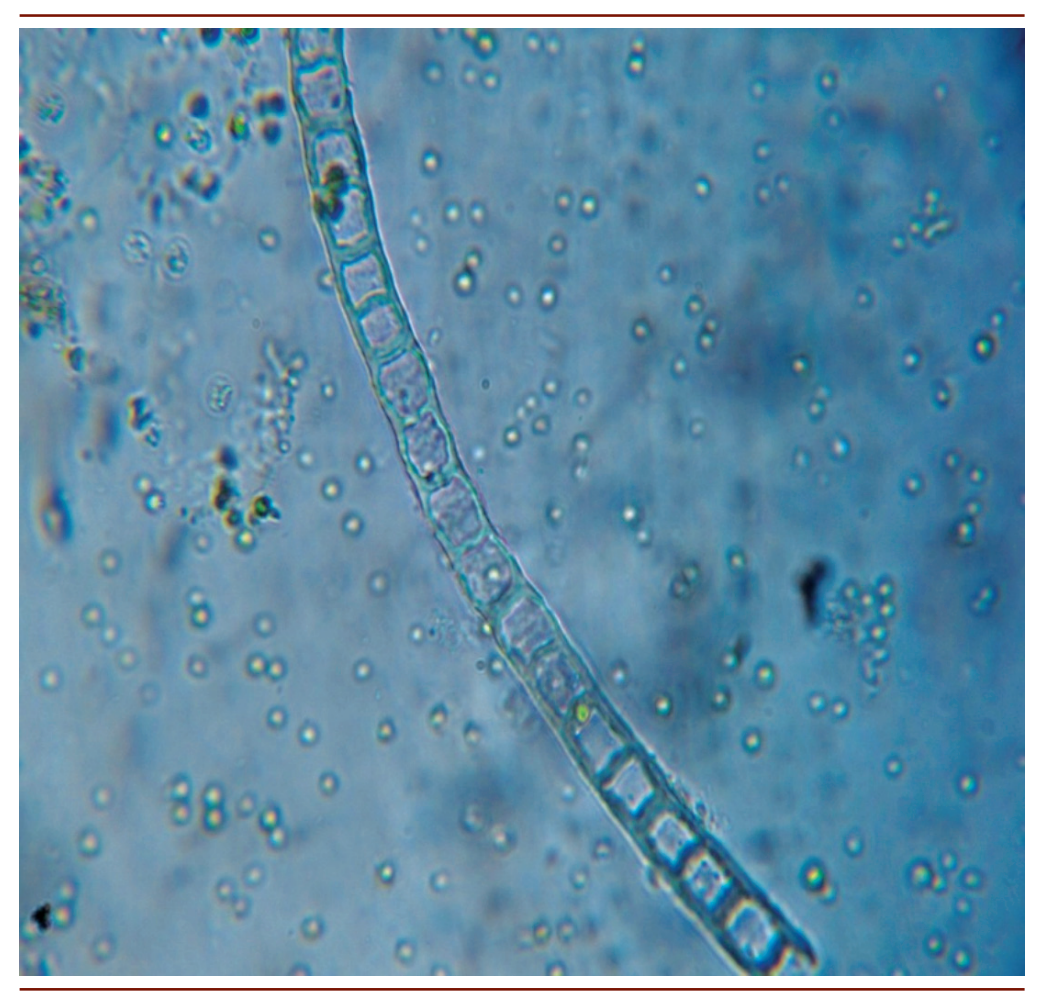

Microspora sp. (40x)

Figura 8. Microspora sp., Rabenhorst L (1868). Observado solo en el sector de "Aguas Hediondas", Tufiño, provincia del Carchi, Ecuador.

Tanto Dictyosphaerium sp., como en muchos de los cultivos envejecidos Microspora sp., fueron observadas solo en un sitio; ya que aparentemente su hábitat está asociado a ambientes de ese sector con abundantes aguas sulfurosas. No obstante, entre las cianobacterias se destaca Leptolyngbya sp., por ser la más distribuida en todas las localidades muestreadas (Figura 5 y 6), lo cual sugiere su capacidad adaptativa, incluso en condiciones estresantes de laboratorio, en cuanto a limitación de nutrientes, temperatura e iluminación, incluso al llegar a ser exclusiva (Abazari et al., 2013).

Según Anagnostidis y Komárek (1988), Leptolyngbya se define como un clúster comprendido por los géneros tradicionales: Lyngbya, Phormidium y Plectonema (LPP grupo B). Las especies de este género (Figura 9) presentan filamentos delgados (0.5 a $3.5 \mu \mathrm{m})$, tilacoides parietales y con vainas facultativas. Varias especies son formadoras de clústeres o biofilms (Komárek, 2007; Albertano y Kováčik 1994). 


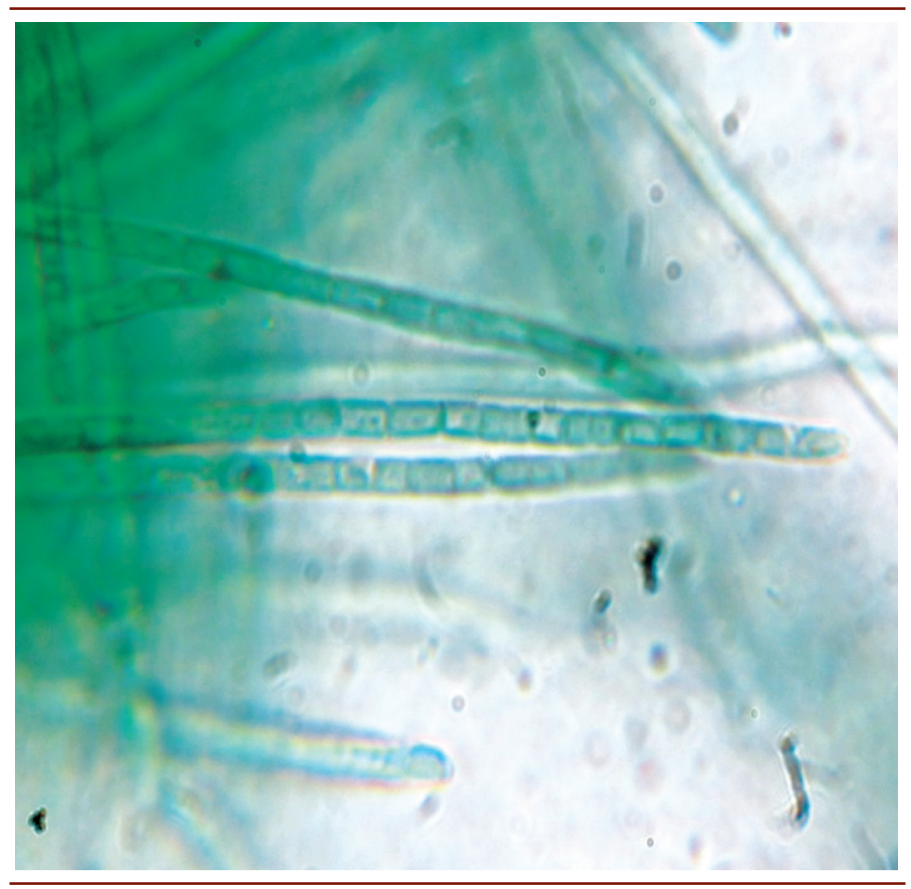

Leptolyngbya sp. (100x)

Figura 9. Leptolyngbya sp., Anagnostidis y Komárek (1988), cianobacteria con mayor frecuencia observada en consorcios y en cultivos aislados.

\section{DISCUSIÓN}

La fase de bioprospección de cepas de microalgas y de cianobacterias para ser incluidas en colecciones o banco de germoplasma, constituye la primera fase de exploración de aquellos ambientes naturales que pueden contener una rica biodiversidad o simplemente permite la detección de estos microorganismos en sitios únicos como de carácter endémico o asociados a otros mediante consorcios los cuales también representan un potencial para investigación desde el punto de vista ecológico, taxonómico, biotecnológico y ambiental.

Algunos de los hábitats analizados presentan características particulares y condiciona la adaptabilidad de las diversas especies a los mismos. En este caso, al menos, la cepas de Navicula sp., Cymbella sp., Diyctyosphaerium sp., Microspora sp., y Mougeotia y las cianobacterias: Phormidium sp. y Leptolyngbya sp., encontradas en aguas termales de "San Vicente", Santa Elena y de "Aguas Hediondas", Carchi pueden ser consideradas de ambientes extremos.

La mayor riqueza específica observada en diatomeas y clorofitas parece indicar su posible adaptación a los distintos nichos ecológicos. Mientras que, las cianobacterias suelen ser altamente competitivas ante la deficiencia de nitrógeno y otros elementos, como en el caso de Nostoc sp., Calothrix sp., Anabaena sp. y Leptolyngbya sp. Además, de presentar elevada capacidad de adaptación a ambientes extremos de $\mathrm{pH}$, deficiencia en nutrientes, altairradiación solar, condiciones anóxicas, aguas sulfurosas, aguas eutróficas 
y alcalófilas (Vincent, 2007). Entre las estaciones de muestreos que presentan condiciones extremas de $\mathrm{pH}$, temperatura, aguas sulfurosas y condiciones anóxicas se encontraron en el Balneario de "Aguas Hediondas", Carchi y en los baños termales de "San Vicente", Santa Elena.

La elevada frecuencia de Chlorella sp., Desmosdesmus sp. y Scenedesmus sp. y de Leptolyngbya sp., en condiciones de laboratorio, confirma su alta adaptabilidad fisioecológica con respecto a otros microorganismos en consorcios. Además, llegaron a mantenerse exclusivas en muestras cultivadas durante el período de estudio, lo cual les confiere la susceptibilidad de ser aisladas rápidamente. Chlorella sp. y Desmosdesmus sp., logran surgir como organismos aislados a partir de consorcios, probablemente debido a la producción de compuestos inhibitorios para otros microorganismos. En cultivo, la competencia ecológica de Chlorella vulgaris mediante alelopatía sobre Pseudokirchneriella subcapitata ha sido descrita lo cual demuestra el efecto inhibitorio de chlorelina (Fergola et al., 2007).

También se demuestra que a partir de colectas de diferentes localidades, se establece una sucesión de especies, conforme van cambiando las condiciones de los cultivos en relación con la disponibilidad de nutrientes, temperatura, flora bacteriana asociada, iluminación, $\mathrm{pH}$. Se pudo determinar que en muestras colectadas de laguna de Yahuarcocha y lago de San Pablo, Imbabura, se observó un predominio de diatomeas (Navicula sp., Epithemia sp., Diatoma sp., Gomphonema sp., Synedra sp., Nitzschia sp. y Melosira sp.) al inicio de la evaluación; pero luego fueron sustituidas por clorofitas (Chlorella sp., Desmodesmus sp. y Scenedesmus sp.) y en muchos casos por Leptolyngbya. Velasco et al., (1984), al realizar observaciones mensuales en un cultivo mixto o en consorcio, describen la sucesión de especies con un predominio de Niztschia sp., luego sucedida por Scenedesmus sp., y por último una preponderancia de Chlorella sp.

La competencia se hace más evidente cuando a los cultivos de colección no se les suministra nutrientes periódicamente. Es por ello que se ha demostrado dominancia de algunas sobre otras y así pueden aparecer completamente aisladas, como producto de estas interacciones competitivas. En un estudio realizado sobre la influencia de la deficiencia de $\mathrm{N}$ y $\mathrm{P}$ en las interacciones competitivas entre Chlorella vulgaris $\mathrm{y}$ Scenedesmus acutus y de acuerdo con los modelos de competencia de Lotka-Volterra, se evidenciaron tres situaciones diferentes: exclusión competitiva de $S$. acutus por $C$. vulgaris en el tratamiento control; exclusión de alguna de las dos y la de coexistencia en el tratamiento con deficiencia de fósforo (González, 2010). En contraposición, en otras cepas de clorofitas, puede no ocurrir el mismo efecto y en este sentido se ha descrito coexistencia entre Chlorella sp., y Chlorococcum sp., en cultivos limitados en $\mathrm{N}, \mathrm{P}$ y Fe; con lo cual se sugiere que las interacciones entre ambas especies no es afectada por ningún factor alelopático (Dow 
et al., 2006). Es posible que, el predominio de las cepas de Chlorella sp., Desmodesmus sp. y Scenedesmus sp., observadas, pueda deberse a la producción de compuestos alelopáticos; de tal manera que logren excluir a los demás microorganismos existentes previamente en los consorcios.

Tales estudios conllevan recomendar que durante el proceso de mantenimiento de consorcios en colecciones se deben monitorear periódicamente la riqueza específica en estos, así como seleccionar medios de cultivos selectivos para promover el crecimiento específico de cepas seleccionadas en función de sus requerimientos nutricionales.

En tanto la frecuencia de aparición de Leptolyngbya sp. (13\% de los cultivos aislados y en $13.3 \%$ de los consorcios mixtos microalga-cianobacteria), sugiere también un elevado nivel competitivo de esta cianobacteria frente a otros microorganismos presentes en las muestras. Al respecto, Van der Grinten et al. (2004) al evaluar el efecto del fosfato en biofilms constituidos por las diatomeas, Melosira varians, Nitzschia perminuta, Navicula trivialis y Achnanthes lanceolata y por las cyanobacterias, Leptolyngbya foveolarum y Cylindrospermum stagnale, demostraron que a diferentes concentraciones $(0.5,5.0$ y $50.0 \mu \mathrm{M})$, en condiciones de laboratorio, L. foveolarum y en menor proporción, Nitzschia perminuta, predominaron a mayor concentración de fosfato, facilitado por el crecimiento expansivo y la excreción de inhibidores generados por L. foveolarum; por lo tanto, se postula como una cianobac- teria con estrategia ecológica ante altas concentraciones de fosfato.

Así mismo, Van der Grinten et al. (2005), describen el efecto de la luz, temperatura, $\mathrm{pH}$ y nutrientes sobre un biofilm con Leptolyngbyafoveolarumy Nitzschiaperminuta y encuentran inhibición del crecimiento de la diatomea por parte de la cianobacteria, tanto a baja como a elevadas intensidades luminosas, incremento del pH y limitación de nutrientes.

Tales resultados, posiblemente, permitan explicar la elevada frecuencia de aparición de Leptolyngbya tanto en consorcios como en los cultivos donde resultó el único el microorganismo en perdurar; mientras no se le adicionaba medio de cultivo fresco a las muestras.

Por lo tanto, se recomienda realizar estudios sobre la ecofisiología de las diversas especies de Leptolyngbya, por cuanto se ha observado crecimiento de la misma en cultivos masivos de microalgas y en cultivos mantenidos en laboratorio. Además, podría ser utilizada para ficorremediación de efluentes $y$ para formación de biofilms o de tapetes de sumo interés para biorremediación y acondicionamiento de suelos (Olguín y Sánchez-Galván, 2012; Soltani et al., 2012).

En relación con Dictyosphaerium sp. y Microspora sp., halladas en las aguas termales de Aguas Hediondas, se destacan por ser exclusivas de la zona y posiblemente Dictyosphaerium sp., aún no han sido 
reportadas para Ecuador (Gallo y Apolo, 2012), por lo cual amerita realizar en dicha área más estudios de bioprospección de microorganismos que puedan presentar interésenbiotecnologíaocomobioindicadores de zonas sulfurosas y/o termófilas.

Las microalgas y cianobacterias aisladas, así como las mantenidas en consorcios, conforman un recurso biológico para investigación en biodiversidad y biotecnología; de allí el reto de conservar la colección de cultivos de forma sustentable, en medios de cultivo tanto líquidos como sólidos, según las condiciones óptimas de luz y temperatura, bien sea en cámaras de cultivo o a temperatura ambiente (Belkinova et al., 2002).

El estudio realizado formaliza la metodología para detectar las diferencias en cuanto a riqueza específica, permanencia, abundancia, sucesión de taxa, durante el proceso de aislamiento; adicionalmente permite recomendar que las muestras originales deben ser mantenidas como fuentes de reclutamiento de microorganismos.

\section{CONCLUSIONES}

- Chlorella sp. y Desmodesmus sp., resultaron los taxa más susceptibles de ser aislados, seguidos de Leptolyngbya sp. Se identificaron sitios de muestreos con elevada diversidad, tales como laguna "El Salado", San Gabriel, Carchi, laguna "Yahuarcocha" y lago "San Pablo", Imbabura y uno con exclusividad de taxa balneario de "Aguas Hediondas", Carchi, en el cual se encontró Dictyosphaerium sp.,
Microspora sp. y Mougeotia sp.

- Se demuestra que los consorcios clasificados en microalga-microalga, cianobacteria-cianobacteria y microalgacianobacteria, constituyen microsistemas biológicos que deben ser conservados en las colecciones de cultivos.

- Chlorella sp. y Desmodesmus sp., fueron las microalgas más susceptibles de ser aisladas dadas sus capacidades de adaptación a condiciones de laboratorio. Mientras que la cianobacteria Leptolyngbya demostró ser altamente competitiva, adaptándose en cultivos deficientes en nutrientes, envejecidos y deshidratados.

\section{AGRADECIMIENTOS}

A la Escuela de Bioanálisis de la Pontificia Universidad Católica del Ecuador por haber facilitado las condiciones para la realización de la presente investigación y por ser la depositaria de la colección de microalgas y cianobacterias. Este trabajo ha sido financiado por el Proyecto Prometeo de la Secretaría Nacional de Educación Superior Ciencia, Tecnología e Innovación (Ecuador).

\section{REFERENCIAS BIBLIOGRAFICAS}

Abazari M, Zarrini G y Rasooli I. 2013. Antimicrobial Potentials of Leptolyngbya sp. and Its Synergistic Effects With Antibiotics. Jundishapur Journal of Microbiology, 6(5): e6536. 
Albarracín I, Cravero M, Salomón R, Pío G, OtañoS, MorisonS, Parra A y Prósperi C. 2009. Desarrollo del laboratorio de microalgas en Patagonia Argentina. Facultad de Ciencias Naturales. UNPSJB Trelew-Chubut. Argentina.

Albertano P y Kováčik L. 1994. Is the genus Leptolyngbya (Cyanophyte) a homogeneous taxon? Archiv für Hydrobiologie/Algological Studies, 75: 37-51.

Anagnostidis K y Komárek J. 1988. Modern approach to the classification system of cyanophytes. 3-Oscillatoriales. Archiv für Hydrobiologie/Algological Studies, 50-53: 327-472.

Arredondo Vega BO. 1997. Colección de microalgas. Centro de Investigaciones Biológicas del Noroeste, S. C. (CIBNOR). 22-23. CONACyT. México DF. México.

Australian National Algae Culture Collection (ANACC). 2012. CSIRO Microalgae Supply Service. 2010. Página de Internet: www.csiro. au/ en / Organisation-Structure / National-Facilities / AustralianNational-Algae-Culture-Collection Consultada junio-2013.

Banco Español de Algas (BEA). 2008. Proyecto: Microalgas. Subproyecto: Creación y Consolidación del Banco Español de Algas (BEA). Universidad de Las Palmas de Gran Canaria. España.
Belkinova D, Kirjakov I y Mladenov R. 2002. Catalogue of the strains of Plovdiv Algal Culture Collection (PACC), Trav. Sci. Univ. Plovidv, Plantarum, 38(6): 3-78

Billard C. 1987. L'algothèque du laboratoire d'algologie fondamentale et appliquée de l'Université de Caen. Cryptogamie Algologie, 8(2), 79-90.

Bourrelly P. 1981. Les Algues d'Eau Douce. Tome I. Les Algues Vertes. Second Edition. N. Boubée et Cie, Paris. 576 pp.

Buitrago E, Llano M y Frontado K. 1999. El banco de cepas de microalgas de la Estación de Investigaciones Marinas de Margarita, Fundación La Salle de Ciencias Naturales. Venezuela. MEMORIA. Fundación La Salle de Ciencias Naturales. Tomo LIX, 151: 67-73.

Castree N. 2003. Bioprospecting: from theory to practice (and back again). Transactions of the Institute of British Geographers, 28(1): 35-55.

Delgadillo I, Góngora F y Medellín F. 2009. La colección de microalgas de la Universidad Pedagógica Nacional como estrategia didáctica para la enseñanza y aprendizaje de conceptos biológicos. Número Extraordinario. $4^{\circ}$ Congreso Internacional sobre Formación de Profesores de Ciencias. Colombia. 
Dow AR, Gilliand JL, Raymond RAyShiring JJ. 2006. Competition between Chlorella sp. and Chlorococcum sp.: a study in nutrient limitation. Journal of Ecological Research, 8:20-25.

Eaton AD, Clesceri LS, Greenberg AE, Franson MAH. 1995. Key for identification of freshwater algae commun in water supplies and polluted water. En Standar Methods for the Examination of water and wasterwater. 19th Ed. American Public Health Association. Washington.

Fergola M, Cerasuolo A, Pollio Pinto G y DellaGreca M. 2007. Allelopathy and competition between Chlorella vulgaris and Pseudokirchneriella subcapitata: Experiment and mathematical model. Ecological Modelling, 208: 205-214.

Fernández-Concha GC., Sosa V, León de la Luz JL. y León-Cortés J. 2004. Colecciones biológicas de los centros de investigación CONACyT-México DF. México.

Friedl T y Lorenz M. 2012. The culture collection of algae at Göttingen University (SAG): a biological resource for biotechnological and biodiversity research. Procedia Environmental Sciences, 15: 110-117.

Gallo N y Apolo B. 2012. Fundamentos de Ficología. Universidad Central del Ecuador. Quito, Ecuador: 120 pp.
García G. 2011. Memoria Anual de Productividad. Banco Español de Algas. Universidad de las Palmas de Gran Canaria, España.

GBIF. 2009. Nodo Nacional de Información de Biodiversidad. Informe de colecciones y bases de datos de biodiversidad en España.

González L. 2010. Influencia de la deficiencia de nitrógeno y fósforo en las interacciones competitivas entre Chlorella vulgaris y Scenedesmus acutus. Tesis de Magister Scientarium en Ciencias Biológicas, Universidad Nacional de Colombia, Bogotá, Colombia.

González MA, Cifuentes AS, Inostroza I y Gómez PI. 2008. Colección de microalgas. Departamento de Botánica, Facultad de Ciencias Naturales y Oceanográficas, Universidad de Concepción, Concepción, Chile.

González MA, Parra O y Cifuentes AS. 1995. Técnicas de cultivo de microalgas en laboratorio. En: Manual de Métodos Ficológicos. Alveal K, Ferrario ME, Oliveira EC y Sar E. (eds.), Universidad de Concepción, Chile. pp 219-250.

Guiry MD. En Guiry MD y Guiry GM. 2013. Algae Base. World-wide electronic publication. National University of Ireland, Galway. http: / / www.algaebase.org 
Komárek J. 2007. Phenotype diversity of the cyanobacterial genus Leptolyngbya in the maritime Antarctic. Polish Polar Research, 28 (3): 211-231.

Komárek J y Anagnostidis K. 2005. Teil: Cyanoprokaryota-2. Teil/2nd Part: Oscillatoriales En Büdel B, Krienitz L, Gärtner G y Schagerl M. (eds). Süßwasserflora von Mitteleuropa. 19/2. Elsevier/Spektrum, Heidelberg. 759 pp.

Komárek J y Anagnostidis K. 1989. Modern approach to the classification system of cyanophytes. 4. Nostocales. Archives für Hydrobiology Suppl./ Algological Studies. 82: 247-345.

Krammer K y Lange-Bertalot H. 1991. Bacillariophyceae. 1. Naviculaceae. En Ettl H, Gerlfoff J, Heynig H y Mollenhauer D. (eds). Süßwasserflora von Mitteleuropa, Band 2/1. Gustav Fischer Verlag: Sttugart, New York. 876 pp.

Krienitz L, BocK C, Kotut K y Pröschold T. 2012. Genotypic diversity of Dictyosphaerium-morphospecies (Chlorellaceae, Trebouxiophyceae) in African inland waters, including the description of four new genera. Fottea, 12(2): 231-253.

Krienitz L, Bock C, Luo W y Pröschold T. 2010. Polyphyletic origin of the Dictyosphaerium morphotype within Chlorellaceae (Trebouxiophyceae). Journal of Phycology, 46(3): 559-563.
Lascuráin M, List R, Barraza L, Díaz Pardo E, Gual Sill F, Maunder M, Dorantes J y Luna VE. 2009. Conservación de especies ex situ. En Comisión Nacional para el Conocimiento y Uso de la Biodiversidad. Capital Natural de México. Estado de Conservación y Tendencias de Cambio. Conabio, 2(12): 517-544, México.

López-Adrián S y Barrientos-Medina RC. 2005. La colección de microalgas dulceacuícolas y marinas de la Península de Yucatán. Ediciones de la Universidad Autónoma de Yucatán. Mérida: 55 pp. México.

Montes de Oca N, González R, Riverón Y, Núñez A, Villoch A y Rodríguez N. 2008. Establecimiento y desarrollo de la colección de cultivos del CENSA. Rev. Salud Animal, 30(1): 17-24.

Mora López M. 2010. Evaluación de la potencialidad biotecnológica y biodiversidad de microorganismos fotosintéticos extremófilos de Costa Rica. Proyecto-062-07. Universidad de Costa Rica.

Olguín EJ y Sánchez-Galván G. 2012. Heavy metal removal in phytofiltration and phycoremediation: the need to differentiate between bioadsorption and bioaccumulation. New Biotechnology, 30(1): 3-8.

Ortega MM. 2009. Herbario Ficológico. Facultad de Biología, Universidad 
Michoacana de San Nicolás de Hidalgo, México.

Rumrich U, Lange-Bertalot $\mathrm{H}$ y Rumrich M. 2000. Diatoms of the Andes from Venezuela to Patagonia/ Tierra del Fuego and two additional contributions. In: Lange-Bertalot, $\mathrm{H}$. (ed.), Iconographia Diatomologica. Annotated Diatoms Micrographs. Vol. 9. Phytogeography-DiversityTaxonomy. Koeltz Scientific Books, Königstein, Germany. 672 pp.

Santos L. 2008. Algoteca de la Facultad de Ciencias y Tecnología de la Universidad de Coimbra (FCTUC). Portugal.

Serrano A, Mateo P y Perona E. 2004. Estructura y composición de la comunidad de cianobacterias bentónicas de un arroyo de montaña mediterráneo, el arroyo Mediano (Madrid). Limnética, 23(1-2): 83-94.

Soltani N, Baftechi L, Dezfulian M, Shokravi S y Alnajar N. 2012. Molecular and Morphological Characterization of Oil Polluted Microalgae. International Journal of Environmental Research, 6(2): 481-49.

Starr R. 2010. The Culture Collection of Algae at the University of Texas at Austin (UTEX).

Stein JR. 1973. Handbook of Phycological Methods: Culture Methods and
Growth Measurements. Cambridge University Press, Cambridge, England: 448 pp.

Van der Grinten E, Janssen APHM, De Mutsert K, Barranguet C y Admiraal W. 2005. Temperatureand light-dependent performance of the cyanobacterium Leptolyngbya foveolarum and the diatom Nitzschia perminuta in mixed biofilms. Hydrobiologia, 548(1-3): 267-278.

Van der Grinten E, Janssen APHM, Simis SGH, Barranguet C y Admiraal W. 2004. Phosphate regime structures species composition in cultured phototrophic biofilms. Freshwater Biology, 49(4): 369-381.

Velasco Díaz JL, Álvarez Cobelas M, Colmenarejo Morcilla JM, Bustos Aragón A. y Parra Sánchez-Bravo F. 1984. Estudio de laboratorio sobre algas aisladas a partir de aguas residuales urbanas. Secretariado de Publicaciones. Universidad de Murcia. España. Anales de Biología, 2: 191-195.

Vergara C. 2009. Descripción de métodos de recolección y conservación de algas. Herbario de la Universidad de Panamá.

Vincent W. 2007. Cold tolerance in cyanobacteria and life in the cryosphere. En: Seckbach J (ed.) Algae and cyanobacteria in extreme environments, Springer. Heidelberg: 289-304. 